DOI: 10.12957/demetra.2016.22542

\title{
Composição corporal em mulheres transgênero vivendo com HIV/Aids: uma discussão das transformações que interferem na avaliação do estado nutricional
}

\section{Body composition in transgender women living with HIV/Aids: a discussion of changes that impact on the assessment of nutritional status}

\author{
Juliana Lauar Gonçalves' \\ Cláudia Santos de Aguiar Cardoso \\ Adriana Costa Bacelo \\ Raquel Espírito Santo \\ Cristiane Fonseca de Almeidal \\ Paula Simplício da Silva \\ Patrícia Dias de Brito
1 Fundação Oswaldo Cruz, Instituto Nacional de Infectologia Evandro Chagas. Rio de Janeiro-RJ, Brasil.
Apoio financeiro: 0 presente trabalho foi financiado através de concessão de bolsa de pós-doutorado da cooperação CAPES-FIOCRUZ, do programa Brasil sem Miséria. A CAPES não tem qualquer responsabilidade com a concepçãa ou escrita do manuscrito. \\ Correspondência / Correspondence \\ Juliana Lauar Gonçalves \\ E-mail: juliana.lauar@ini.fiocruz.br
}

\section{Resumo}

A prática clínica no atendimento de mulheres transgênero vivendo com HIV/Aids despertou o olhar sobre as consequências dos procedimentos para a adequação ao gênero autodeterminado no processo de avaliação da composição corporal. Mulheres transgênero são pessoas que, ao nascer, receberam a atribuição de homem, mas se autoidentificam e reivindicam o reconhecimento social e legal como mulheres. Em busca da feminilização, podem vir a fazer uso de géis de silicone, de terapia hormonal ou até mesmo de procedimentos nocivos, como injeção de óleo de silicone. Esses procedimentos resultam em alterações antropométricas e modificações dos compartimentos corporais, como o tecido adiposo subcutâneo, massa óssea e massa magra. Ocorrem também, em alguns casos, especificidades, como a redução do volume prostático e o desenvolvimento lobular e acinar das mamas. O panorama é dificultado ainda no contexto da infecção pelo HIV, cujo tratamento antirretroviral, em alguns casos, tem como reação adversa a lipodistrofia. A lipodistrofia é caracterizada por alterações na distribuição de gordura corporal (lipoatrofia e lipo-hipertrofia) e deve ser considerada na avaliação da composição corporal dessa população. Diante da grande vulnerabilidade de mulheres transgênero pela infecção com o HIV, a OMS recomenda que sejam adotadas medidas específicas de prevenção, tratamento e serviços de saúde para essa população. Portanto, no presente trabalho pretendemos iniciar a reflexão sobre os desafios para a avaliação nutricional de mulheres transgênero vivendo com HIV/Aids.

Palavras-chave: Transgênero. Silicone. Lipodistrofia. Composição corporal, HIV. 


\section{Abstract}

The clinical practice in care of transgender woman living with HIV/Aids has drawn attention to the consequences of gender transformation procedures in the evaluation of body composition. Transgender women are people who were attributed to be men at birth, but identify themselves as women and claim for the social and legal recognition as women. In seeking for feminization, these people are likely to make use of silicone gels, hormone therapy or even harmful procedures such as silicone oil injection. These procedures result in anthropometric and body compartment changes such as observed in subcutaneous adipose tissue, bone and lean body mass. In some cases, there is the occurrence of prostatic volume reduction and development of lobular and acinar breasts. In the context of HIV infection, this scenario is even more difficult to handle, since the treatment with antiretroviral, in some cases, could generate adverse reaction such as lipodystrophy. Lipodystrophy is characterized by changes in body fat distribution (lipoatrophy and lipohypertrophy) and must be considered in the body composition evaluation of this population. Because of the considerable vulnerability of transgender women with HIV infection, the World Health Organization recommends the adoption of specific actions of prevention, treatment and health care for this population. Therefore, in the present work, we intend to start thinking about the challenges to nutrition evaluation of transgender women living with HIV/Aids.

Key words: Transgender. Silicone. Lipodystrophy. Body composition, HIV.

\section{Introdução}

Recentemente, a OMS reconheceu a vulnerabilidade diferenciada de mulheres transgênero à infecção pelo HIV, cerca de 50 vezes maior que a população masculina ou feminina cisgênero, e recomendou que fossem adotadas medidas específicas de prevenção, tratamento e na prestação de serviços de saúde., ${ }^{1,2}$

Transgênero é a palavra-chave para as pessoas cuja identidade de gênero e sua expressão não estão em conformidade com as normas e expectativas tradicionalmente associadas com o gênero que lhes foi atribuído à nascença. Fazem parte dessa denominação pessoas que são transexuais, transgênero ou não conformes de outra forma considerada gênero. ${ }^{2}$ A expressão do gênero pode ocorrer de maneiras variadas, como masculino, feminino e andrógino (em que há traços e comportamentos de gênero imprecisos). Existe também a denominação de gênero 
fluido, em que o indivíduo polariza pelo feminino e masculino em momentos alternados do seu cotidiano. Pessoas transgênero podem se autoidentificar como mulher transgênero, mulher trans ou homem trans, transexual, ou por uma variedade de termos culturais específicos, tais como Hijra (Índia), Kathoey (Tailândia); Waria (Indonésia), ou uma das muitas identidades transexuais. ${ }^{2}$ Para fins de compreensão do presente artigo, utilizaremos a definição de mulher transgênero para a pessoa que, ao nascer, recebeu a atribuição de homem, mas se autoidentifica como mulher. Outra denominação utilizada no presente artigo é o termo cisgênero, que se refere às pessoas cujo gênero autodeterminado é o mesmo que o designado ao nascimento. No contexto da assistência à saúde no Brasil, muito se discute sobre essa temática e desde 2009 é assegurado o direito ao uso do nome social por pessoas transgênero. ${ }^{3}$

Entre os serviços de saúde, a assistência nutricional é reconhecida como área estratégica, capaz de reduzir o risco de agravos e a mortalidade em pessoas vivendo com HIV/Aids. Inicialmente, a desnutrição e a deficiência de nutrientes eram o foco da assistência nutricional a este grupo de indivíduos. Com a indicação e o uso cada vez mais precoce de antirretrovirais, a desnutrição ficou limitada a alguns grupos específicos, como indivíduos com má adesão à terapia antirretroviral, além daqueles que experimentam situações de vulnerabilidade social, como usuários de drogas. ${ }^{4-6}$ Hábitos alimentares inadequados e sedentarismo, associados ao medo do emagrecimento, característico da doença, levaram ao excesso de peso nas pessoas vivendo com HIV/Aids. ${ }^{7}$ Ainda, como um efeito colateral do uso da terapia antirretroviral, esses indivíduos podem desenvolver alterações da composição corporal, com perda ou deposição ectópica de tecido adiposo, denominadas genericamente de lipodistrofia. ${ }^{5}$

A avaliação nutricional é um dos componentes da assistência em nutrição e entre seus métodos, utiliza a mensuração dos compartimentos corporais em diferentes níveis. Pode-se citar, como parte da avaliação nutricional, a determinação do índice de massa corporal (IMC), a mensuração de circunferências corporais e de dobras cutâneas e a determinação de massa gorda e massa livre de gordura pela técnica de bioimpedância elétrica. Uma vez aferidos, esses parâmetros são comparados a valores de referência e classificados por pontos de corte que são previamente definidos para a população e que, em alguns casos, são estabelecidos também em função do gênero. ${ }^{8}$

Em busca de adequação ao gênero autodeterminado, mulheres transgênero podem optar por se submeter a intervenções cirúrgicas ou não cirúrgicas, das quais podemos elencar a cirurgia de redesignação sexual, a tireoplastia, o implante de géis de silicone, a ressecção de costelas flutuantes, a lipoaspiração, além de procedimentos como a terapia hormonal. Algumas dessas intervenções são capazes de gerar alterações corporais importantes, criando assim novos parâmetros métricos que dificultam a comparação dessa população dentro dos pontos de corte existentes para a população cisgênero masculina e feminina. A soma dessas alterações àquelas provocadas pela lipodistrofia aumenta a complexidade da avaliação da composição corporal. ${ }^{9}$ 
Portanto, o presente artigo se propõe a refletir sobre as alterações de composição corporal, com finalidade de conformação ao gênero autodeterminado, como também as causadas pela terapia antirretroviral do HIV em mulheres transgênero vivendo com HIV/Aids. Em nosso entendimento, este é o primeiro trabalho que se propõe a tal reflexão.

\section{Metodologia}

A presente pesquisa se enquadra no campo da diversidade de gêneros com enfoque na atenção à saúde de mulheres transgênero infectadas pelo HIV. Trata-se de um estudo qualitativo realizado a partir da análise dos fenômenos inerentes ao atendimento nutricional dessas mulheres, descrito sob a perspectiva do nutricionista, no âmbito hospitalar e ambulatorial do Instituto Nacional de Infectologia Evandro Chagas (INI).

O Instituto Nacional de Infectologia Evandro Chagas (INI) é uma unidade da Fundação Oswaldo Cruz (FIOCRUZ) que realiza assistência e pesquisa clínica em doenças infecciosas. São atendidos pacientes adultos e idosos, com HIV/Aids, infecção pelo HTLV (vírus T-linfotrópico humano), doença de Chagas, leishmaniose, tuberculose, doenças febris agudas, entre outras. No INI, o atendimento aos pacientes é realizado de forma multiprofissional, objetivando uma atenção integral às necessidades dos mesmos, sendo desenvolvido nos seguintes setores: Ambulatório, Hospital Dia e Centro de Internação Hospitalar.

O Serviço de nutrição tem como objetivo realizar o atendimento nutricional especializado e promover educação nutricional de pacientes visando à promoção, manutenção e recuperação da saúde. A rotina do atendimento nutricional inclui realização de anamnese clínica e alimentar e de medidas antropométricas, checagem de exames laboratoriais, planejamento do tratamento nutricional e orientação nutricional ao paciente.

O atendimento realizado por este Serviço, bem como pelos demais profissionais do INI, é norteado pelo Sistema Único de Saúde e pela concepção ampliada de saúde descrita na Lei Orgânica da Saúde:

A saúde tem como fatores determinantes e condicionantes, entre outros, a alimentação, a moradia, o saneamento básico, o meio ambiente, o trabalho, a renda, a educação, o transporte, o lazer e o acesso aos bens e serviços essenciais; os níveis de saúde da população expressam a organização social

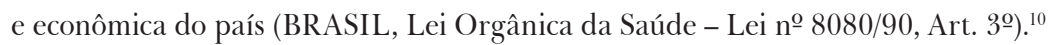

Para a reflexão sobre o tema, realizou-se levantamento bibliográfico sistematizado de estudos nas bases de dados Pubmed e Scielo, sobre questões relativas à composição corporal de mulheres transgênero. Os descritores utilizados na pesquisa foram: "transgenderpersons", "bodycomposition", 
"silicone oils", "silicone gels", "HIV", de acordo com os descritores em ciências da saúde (DeCs). Como critério de inclusão, foram selecionados os trabalhos que relacionavam as alterações de composição corporal relativas ao processo de conformidade de gênero, às características corporais associadas à infecção pelo HIV e à terapia antirretroviral em mulheres transgênero. Trabalhos sobre mulheres transgênero que não tratavam de aspectos relativos à composição corporal não foram incluídos. Não houve limitação de faixa temporal.

\section{Resultados e Discussão}

$\mathrm{O}$ atendimento de mulheres transgênero em serviços de saúde implica alguns desafios para a equipe multiprofissional que precisam ser ultrapassados. No presente trabalho, propusemo-nos a refletir sobre os fatores que devem ser considerados no atendimento nutricional dessas mulheres. Apesar da grande relevância do tema, ele ainda é negligenciado e é escassa a literatura científica com essa abordagem.

No que tange à avaliação nutricional, objeto do presente artigo, devemos considerar que ainda é desconhecido o efeito final do processo de transformações para a construção do corpo feminino. Os procedimentos de adequação ao gênero são muito amplos e incluem alterações de comportamento, postura, empostação da voz, uso de hormônios, dilatação do canal vaginal e implantes ou injeção de silicone. ${ }^{11}$

No atendimento das mulheres transgênero nos deparamos com a questão de que os parâmetros ditos de normalidade são construídos com base nos gêneros binários. Sendo essas as únicas ferramentas disponíveis para a classificação e diagnóstico corporal dessas mulheres, surge um novo impasse: Em que grau os mesmos devem ser aplicados a indivíduos transgênero? Percebemos em nossa rotina que possivelmente existe um viés na interpretação dos dados. As novas proporções corporais de mulheres transgênero, que realizaram intervenções de feminilização, não se encaixam mais no padrão de homens cis, como também estão inadequadas às proporções cis femininas. Portanto, o diagnóstico baseado nas medidas antropométricas pode identificar equivocadamente o estado nutricional dessas pessoas.

Recomendações recentes do Centro de Excelência para a Saúde de Transgêneros indicam que pessoas que iniciem o processo de readequação física ainda na puberdade, com administração de hormônios, apresentarão menos diferenças corporais em relação aos cisgêneros. ${ }^{12}$ Após a maturação sexual, as transformações para a adequação ao gênero poderão ser mais superficiais, impactando em menor grau nas proporções e aspectos corporais, bem como nas características teciduais. ${ }^{12}$

No Brasil, é notório que o processo de readequação ao gênero na vida adulta é a realidade mais habitual. Impasses sociais, culturais e religiosos permeiam a construção do indivíduo, dificultando 
a autodesignação precoce da identidade de gênero escolhida e também o acesso ao tratamento hormonal. ${ }^{13}$ Em regiões em que a questão de readequação de gênero é mais debatida, como na América do Norte e Europa, é possível que exista maior similaridade entre corpos cis e trans.

Alguns aspectos relevantes a ser considerados na avaliação da composição corporal de mulheres transgênero serão discutidos a seguir.

\section{Utilização de Géis de Silicone para a transformação corporal}

Implantes de géis de silicone são amplamente utilizados para reconstrução e aumento das mamas desde $1960 .{ }^{14}$ Entre mulheres transgênero, um importante percentual opta pelo aumento das mamas através dessa técnica. A literatura ainda é escassa sobre os efeitos de implantes de géis de silicone na avaliação da composição corporal. Um primeiro trabalho foi publicado em 2012, no qual Yamaguchi e colaboradores ${ }^{15}$ tentaram esclarecer se implantes de géis de silicone poderiam interferir na análise da composição corporal por meio da técnica de bioimpedância elétrica. Para isso, foi conduzido um estudo prospectivo com 20 mulheres em que a análise da composição corporal por bioimpedância foi realizada no pré-operatório e dois meses após o implante de géis de silicone. Os autores concluíram que o implante de géis de silicone foi reconhecido como massa gorda. Em relação ao IMC, não houve qualquer variação, uma vez que o peso médio dos implantes foi de 600 gramas. Esse trabalho abre a perspectiva de se considerar a presença de géis silicone para a avaliação da composição corporal por bioimpedância, uma vez que ocorre a superestimação da massa gorda individual.

No entanto, o maior desafio sobre as modificações corporais por mulheres transgênero diz respeito ao uso de óleos de silicone para fins industriais como alternativa estética. Pumping é o termo em inglês para a prática ilegal e potencialmente agressiva de injeção de óleo de silicone diretamente nos tecidos para a feminilização corporal. Geralmente, é realizada em condições sanitárias inadequadas e tornou-se popular entre mulheres transgênero em função do resultado rápido, da alta disponibilidade e do baixo custo. ${ }^{16}$ Mulheres transgênero em todo o mundo têm utilizado essa prática não só para o aumento das mamas, mas também para o remodelamento das nádegas, coxas e face. ${ }^{17}$ Os volumes injetados de forma subcutânea podem ser consideravelmente altos, alterando o peso, as dimensões das circunferências, dobras cutâneas e IMC. No caso de indivíduos que injetam grande volume de óleo de silicone, a utilização dos parâmetros antropométricos fica comprometida, mesmo que se opte pela comparação das aferições do próprio indivíduo ao longo do tempo.

A análise por bioimpedância em indivíduos com injeção de óleo de silicone provavelmente sofrerá a mesma limitação discutida acima, com superestimação da massa gorda. Entretanto, 
existe a possibilidade de não se obter o mesmo resultado, já que o óleo de silicone apresenta propriedades químicas diferente dos géis de silicone, além de não estar encapsulado e ser injetado de forma subcutânea permeando os tecidos. Em relação às complicações mais graves da prática de injeção de óleo de silicone, está o processo inflamatório crônico, que também é a mais comum, com formação de granuloma e fibrose extensiva.

\section{Terapia hormonal e suas consequências para a composição corporal}

Mulheres transgênero muitas vezes recorrem à farmacoterapia como parte do processo de adequação ao gênero autodeterminado. Inicialmente, a paciente utiliza o antiandrogênio para bloqueio da produção e androgênios gonadais (popularmente denominado castração química). ${ }^{19}$ Em seguida, inicia-se o uso de estrogênios que, por sua vez, diminuem a produção de androgênios adrenais em $27 \%$ a $48 \% .^{18} \mathrm{~A}$ administração de estrogênio por via oral aumenta a densidade mineral óssea, mas diminui o turnover ósseo. Reduz também o volume prostático e, em combinação com antiandrogênios, aumenta os níveis séricos de leptina. O tecido adiposo subcutâneo é aumentado e o tecido adiposo visceral é diminuído com o uso do estrogênio oral. Outra alteração importante da farmacoterapia hormonal é o desenvolvimento lobular e acinar das mamas, que são alterações histológicas semelhantes às femininas..$^{19}$

A terapia hormonal é capaz de modificar a composição corporal dessas mulheres transgênero, com alterações máximas em torno de 1 a 3 anos após o início do tratamento. ${ }^{20}$

Em 2008, Lapauw ${ }^{21}$ e colaboradores avaliaram, em um estudo transversal, a composição corporal de 23 mulheres transgênero pelas técnicas de densitometria por raios X (DEXA) e tomografia computadorizada periférica. As voluntárias foram recrutadas pelo menos 3 anos após a cirurgia de redesignação sexual e estavam em uso de farmacoterapia. Como grupo controle foram recrutados 46 homens cisgênero saudáveis, pareados pela altura e idade. Os resultados indicaram uma redução total e regional na massa muscular, além da redução de força do aperto de mão avaliada por dinamômetro comparativamente com o grupo controle. Inversamente, ocorreu aumento total e regional da massa adiposa. O conteúdo e a densidade óssea também foi menor no grupo de mulheres transgênero. Os resultados desse trabalho foram correlacionados com menores níveis de testosterona como também de atividade física.

Outro trabalho, realizado em 2013, avaliou a massa muscular e massa óssea por DEXA em 25 mulheres transgênero antes do início da farmacoterapia e as comparou com um grupo controle de 25 homens cisgênero pareados por idade, além de uma população com 941 homens cisgênero. ${ }^{22}$ Esse trabalhou constatou que esse grupo de mulheres transgênero apresentava redução de massa muscular, força e massa óssea prévia à terapia hormonal comparada ao grupo controle. Os dados foram atribuídos ao nível de atividade física menor entre as mulheres transgênero. 
Em conjunto, esses dados nos indicam que a terapia hormonal também deve ser considerada na avaliação nutricional de mulheres transgênero, uma vez que ela modifica toda a redistribuição dos compartimentos de massa magra e massa gorda e que comparações com homens cisgênero não são adequadas.

\section{Lipodistrofia e os desafios para sua avaliação}

Após a introdução da terapia antirretroviral de alta eficiência, a infecção pelo vírus HIV se tornou uma doença crônica, e alguns comprometimentos de saúde, não necessariamente relativos à patogenia pelo vírus, passaram a ser identificados. ${ }^{23}$

A lipodistrofia do HIV é uma síndrome associada ao uso da terapia antirretroviral, caracterizada pela dislipidemia, com aumento do colesterol e triglicerídeo, aumento da glicemia associada à resistência à insulina e mudanças na redistribuição de gordura corporal. Os sintomas da lipodistrofia relativos à redistribuição da massa gorda envolvem lipoatrofia, devido à perda de gordura subcutânea na face e membros, além da lipo-hipertrofia, na qual existe aumento da adiposidade central. ${ }^{25}$

Os pacientes em uso da terapia antirretroviral, incluindo as mulheres transgênero, podem vir a se submeter a procedimentos estéticos, cirúrgicos ou não, a fim de minimizar as alterações na imagem corporal.

As alterações de imagem corporal são descritas na literatura como importante comprometedor da feminilidade e autoestima, ${ }^{24}$ interferindo negativamente na autoimagem e acarretando problemas psicológicos e sociais, ${ }^{25}$ que influenciam fortemente na qualidade de vida. ${ }^{26}$ Diante desse fato, tratamentos estéticos têm sido considerados importantes estratégias na promoção de saúde emocional dessa população. ${ }^{27}$

No contexto da avaliação nutricional, a lipodistrofia necessita de uma atenção adicional. Um trabalho recente determinou pontos de corte e razões das circunferências corporais para a avaliação da lipodistrofia em homens cisgênero vivendo com HIV/Aids. Nesse trabalho, concluiu-se que o uso de parâmetros antropométricos específicos para essa população é capaz de auxiliar no diagnóstico da lipodistrofia com a finalidade de uma intervenção precoce e mesmo para proteger o indivíduo de disfunções metabólicas. ${ }^{28}$ Apesar de a população analisada ser diferente da tratada no presente artigo, podemos inferir que as modificações geradas pela lipodistrofia devem também ser consideradas nessas mulheres transgênero, além do importante complicador de que essas modificações resultam muitas vezes na necessidade de correções estéticas que, como discutido anteriormente, dificultam a avaliação da composição corporal. 


\section{Considerações finais}

É importante despertar a sensibilidade para compreender a nova realidade da população e repensar formas de avaliação individualizadas que se adaptem melhor às pessoas que são atendidas diariamente em consultórios, hospitais e centros de pesquisa.

A atenção nutricional de mulheres transgênero deve considerar não somente a avaliação física diferenciada, em que os padrões de normalidade binários não viabilizam referência suficiente para a classificação do estado nutricional, mas também as questões relacionadas à autoestima, o que torna mais complexa a pratica clínica do nutricionista.

\section{Referências}

1. van Griensven F, Na Ayutthaya PP, Wilson E. HIV surveillance and prevention in transgender women. Lancet Infectious Diseases 2013; 13(3):185-186.

2. World Health Organization. Policy brief: transgender people and HIV. Geneva: WHO; 2015. 28 p.

3. Brasil. Portaria n0 1.820, Dispõe sobre os direitos e deveres dos usuários da saúde. 2009 ago 13. Diário Oficial da União 14 ago. 2009; 1(155):80.

4. Anema A, Chan K, Chen Y, Weiser S, Montaner JSG, Hogg RS. Relationship between food Insecurity and mortality among HIV-positive injection drug users receiving antiretroviral therapy in British Columbia, Canada. Plos One 2013; 8(5):e61277.

5. Fernandes APM, Sanches RS, Mill J, Lucy D, Palha PF, Dalri MCB. Lipodystrophy syndrome associated with antiretroviral therapy in HIV patients: considerations for psychosocial aspects. Rev Latino-Am Enfermagem 2007; 15(5):1041-5.

6. Ivers LC, Cullen KA, Freedberg KA, Block S, Coates J, Webb P, et al. HIV/AIDS, Undernutrition, and Food Insecurity. Clinical Infectious Diseases 2009; 49(7):1096-102.

7. Jaime PC, Florindo AA, Latorre MRDO, Brasil BG, Santos ECM, Segurado AAC. Prevalence of overweight and central obesity in HIV/AIDS patients treated with highly active antiretroviral therapy. Rev Bras Epidemiol. 2004; 7(1):65-72.

8. Agriculture USD of U.S. Department of Health and Human Services. Dietary guidelines for americans, 2010. 7th ed. Washington, DC: U.S. Government Printing Office; 2010.97 p.

9. Athayde AVL. Transexualismo masculino. Arq Bras Endocrinol Metab. 2001; 45(4):407-14.

10. Brasil. Lei no 8.080. Lei Orgânica do SUS. Diário Oficial da União 19 set. 1990.

11. Petry AR. Transgender women and the gender reassignment process: subjection experiences suffering and pleasure in body adaptation. Rev Gaúcha Enferm. 2015; 36(2):70-5. 
12. Deutsch M, editor. Guidelines for the primary and gender-affirming care of transgender and gender nonbinary people. 2 ed. São Francisco: University of California; 2016. 199 p.

13. Vila S, Grossi MP. Transexualidade e movimento transgênero na perspectiva da diáspora queer. V Congresso da Associação Brasileira de Estudos da Homocultura; 23 set. 2010; Natal, RN. Natal: ABEH; 2010. 17 p.

14. Gampper TJ, Khoury H, Gottlieb W, Morgan RF. Silicone gel implants in breast augmentation and reconstruction. Annals of Plastic Surgery 2007; 59(5):581-90.

15. Yamaguchi CM, Faintuch J, Silva MM, Modolin M, Hayashi SY, Cecconello I. Interference of silicone breast implants on bioimpedance measurement of body fat. Clinical Nutrition 2012; 31(4):574-6.

16. Murariu D, Holland MC, Gampper TJ, Campbell CA. Illegal silicone injections create unique reconstructive challenges in transgender patients. Plast Reconstr Surg. 2015; 135(5):932e-933e.

17. Rettner R. Illegal silicone butt injections cause host of health problems [Internet]. 18 Jun. 2015 [acesso em: 15 abr. 2016]. Disponível em: http://www.livescience.com/51275-silicone-butt-injections-healthproblems.html

18. Polderman KH, Gooren LJ, van der Veen EA. Effects of gonadal androgens and oestrogens on adrenal androgen levels. Clinical Endocrinology 1995; 43(4):415-21.

19. Giestas A, Palma I. Tratamento endócrino no transtorno de identidade de género. ACTA Obstet Ginecol Port. 2012; 6(4):180-7.

20. Leach C. Pharmacotherapy considerations in the management of transgender patients: an alternative viewpoint. Pharmacotherapy: The Journal of Human Pharmacology and Drug Therapy 2016; 36(4):28-29.

21. Lapauw B, Taes Y, Simoens S, Van Caenegem E, Weyers S, Goemaere S, et al. Body composition, volumetric and areal bone parameters in male-to-female transsexual persons. Bone 2008; 43(6):1016-21.

22. Van Caenegem E, Wierckx K, Taes Y, Schreiner T, Vandewalle S, Toye K, et al. Body composition, bone turnover, and bone mass in trans men during testosterone treatment: 1-year follow-up data from a prospective case-controlled study (ENIGI). Eur J Endocrinol. 2015; 172(2):163-71.

23. Alves MD, Brites C, Sprinz E. HIV-associated lipodystrophy: a review from a Brazilian perspective. Ther Clin Risk Manag. 2014; 10:559-66.

24. Brasil. Ministério da Saúde. Recomendações para terapia anti-retroviral em adultos infectados pelo HIV: 2008. Brasília: Programa Nacional de DST e AIDS; 2008.

25. Power R, Tate HL, McGill SML, Taylor C. A qualitative study of the psychosocial implications of lipodystrophy syndrome on HIV positive individuals. Sex Transm Infect. 2003; 79(2):137-141.

26. Plankey M, Bacchetti P, Jin C, Dass-Brailsford P, Gustafson D, Cohen MH, et al. The association of self-perception of body fat changes and quality of life in the Women's Interagency HIV Study. AIDS Care 2013; 25(12):1544-50. 
27. Quintas RCS, França ER, Petribú KCL, Ximenes RAA, Quintas LFFM, Cavalcanti ELF, et al. Treatment of facial lipoatrophy with polymethylmethacrylate among patients with human immunodeficiency virus/acquired immunodeficiency syndrome (HIV/AIDS): impact on the quality of life. Int J Dermatol. 2014; 53(4):497-502.

28. Beraldo RA, Vassimon HS, Aragon DC, Navarro AM, Albuquerque de Paula FJ, Foss-Freitas MC. Proposed ratios and cutoffs for the assessment of lipodystrophy in HIV-seropositive individuals. European Journal of Clinical Nutrition 2015; 69(2):274-78.

Recebido: $18 / 4 / 2016$

Revisado: 01/8/2016

Aceito: 17/11/2016 
